\title{
PREPARATION OF PLASMA POLYMERIZED FILMS FROM CHLOROFLUOROCARBON
}

\author{
KIYOSHI MIYASHITA and JONY SUGO and AKIRA KOJIMA* \\ Department of Chemistry, Gunma Prefectural Industrial Technology \\ Research Laboratory, Gunma 371, JAPAN \\ *Department of Industrial chemistry, Gunma College of Technology, \\ Gunma 371, JAPAN
}

\begin{abstract}
A Chlorofluorocarbon was converted to a solid state substance for the purpose of nontoxic treatment and resource of specific freon waste. Experiments were performed by means of plasma polymerization from $\mathrm{CFCl}_{13}$ (Trichlorotrifluoroethane) and n-hexane mixtures. The maximum weight deposition rate of the film from CFC113 and $\mathrm{n}$-hexane is $1.5 \times 10^{-3} \mathrm{mg} \mathrm{cm}^{-2} \mathrm{~s}^{-1}$ at a two-one weight mixing ratio of $\mathrm{CFC} 113$ and hexane in the flask tank. This deposition rate is about 10 times as large as that from $n$-hexane itself. The $16 \%$ weight amount of gas mixture introduced into the reaction chamber was converted to polymeric film on the lower electrode at the preparation condition. Solidification recovery in the whole chamber was more than $50 \%$. Results of XPS and IR measurements indicate the existence of C-F and $\mathrm{C}-\mathrm{Cl}$ covalent bonds in the films. UV-VIS spectra of the films were significantly different from that of $n$-hexane itself.
\end{abstract}

\section{Introduction}

Chlorofluorocarbon is a type of molecule which is composed of chlorine, fluorine and carbon. It has been widely used as wash reagent for industrial parts and as coolant in refrigerators. However, Chlorofluorocarbon has been linked with the destruction of the earth's ozone layer and global warming. For this reason, chlorofluorocarbon is classified as a specific freon and will be prohibited for use in the world. The collection of waste and currently utilized freon is aggressively being promoted. At the same time, treatment methods that destroy specific freon to nontoxic substance is being investigated. Some methods that destroy specific freon have already been developed [1],[2]. However, these methods aim mainly at destroying the freon, rather than reuse or resource of it. 
A study that attempted to convert a specific freon to a solid state polymeric substance by means of cold plasma has already been reported [3]. The study attempted to convert trichlorotrifluoroethane (CFC113), the most widely used freon, to a polymeric film. CFC113 did not make any deposition film by itself, by cold plasma, but did make films with the effect of ethylene gas addition at high deposition rates. The results of XPS and FT-IR measurements show the existence of carbon, fluorine and chlorine in the film, also chemical bonds of C-F and C-Cl. Furthermore, a electric property was examined.

This study attemps to find a higher deposition rate of the polymeric film and other type of polymeric film from CFC113. The chemical structure of the films were examined by XPS and FT-IR. Ultraviolet and visible spectra were also measured.

\section{Method}

The schematic diagram of the cold plasma generating apparatus used in this work is shown in Fig. 1. The apparatus manufactured by SAMCO International Lab. Ltd., consists of a glass bell jar enclosing two parallel disk electrodes ( SUS dia. 100mm ). Discharge was supplied by a radiofrequency which operates at $13.56 \mathrm{MHz}$ and delivers a maximum power of 200W. Flow rate of the gas was regulated by a needle valve. The chamber pressure was measured by a Pirani vacuum gauge. The ventilated gases from the chamber was treated to be nontoxic by a equipment of halogenide substance cleaner manufactured by Kanto Denka Kogyo Co., Ltd.,

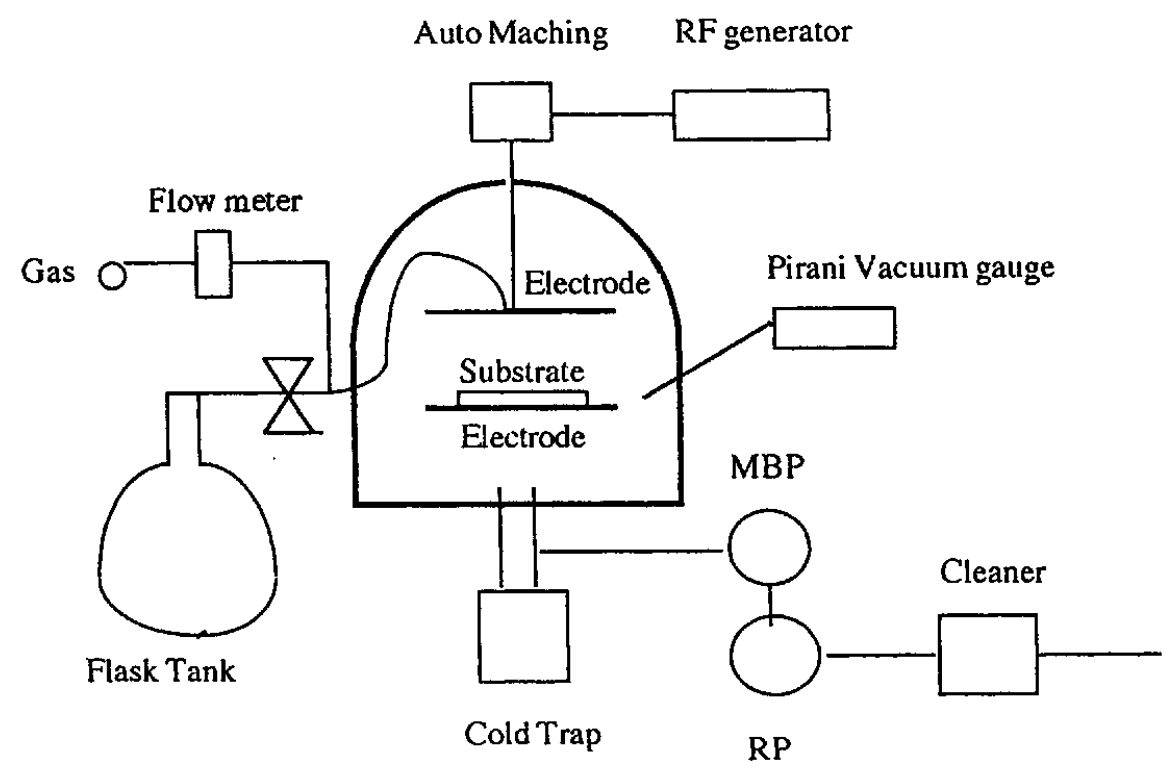

Fig. 1 The schematic diagram of plasma polymerized apparatus 


\section{J. Photopolym. Sci. Technol., Vol.8, No.3, 1995}

Trichlorotrifluoroethane ( $\mathrm{CFCl13}$ ) used in this work was obtain from collected waste freon which were originally provided by Mitsui Fluoro Chemicals. Ltd., The monomer was purified by filtration and evaporation. Additive monomer used as assist gas was n-hexane. The reason why $\mathrm{n}$-hexane was used is because $\mathrm{n}$-hexane is one of hydrocarbon which has a liquid state, like $\mathrm{CFC113}$, at room temperture. It would be easy to introduce the gas mixture into the reaction chamber from a liquid source supplier. These monomers were mixed in a flask tank and lead into the vacuumed reaction chamber and sprayed from holes under the upper electrode. The chamber pressure was regulated to keep at a constant 0.3 Torr by needle valve. Discharge was supplied for three minutes at a power of $100 \mathrm{~W}$. The content of monomers gas in the mixture were quantified by gas chromatography.

Microscope cover glass was used as substrates for deposition. The polymer deposition rates were determined by the weight gained on a microscope cover glass placed on the lower electrode in the plasma induced by discharge for 3 minutes.

The Xray Photoelectron Spectrometer ( XPS ) used was a AXIS-HS equipped with Mg anode Xray source, manufactured by SHIMAZU/KRATOS Co., Ltd., Chemical elements existing in the deposition film were determined by XPS. XPS spectrum of the $\mathrm{C}(1 \mathrm{~s})$ region were deconvoluted into five Gaussian peaks. Fuoreir Transformed Infrared Spectrometer ( FT-IR ) was a Magna-IR 750 manufactured by Nicolet Instrument Co., Ltd., IR measurements were performed by diffuse reflectance method preparing a powder sample collected from the deposition film. Ion chromatography IC500 manufactured by Yokogawa Electric Co., Ltd., was used to detect halogenide ions in the substance trapped in a glass tube cooled by liquid nitrogen. Ultraviolet-visible spectrometer used was UV-VIS228A manufactured by HITACHI Ltd.,

\section{Results and Discussion}

\section{Deposition rates of polymeric films}

As was already reported [3], CFC113 did not make any deposition film on it's own. However, deposition films were obtained by the addition of $n$-hexane. The films have a smooth surface and yellow-brown color. Fig. 2 illustrates the effect of $n$-hexane as a assist gas to $\mathrm{CFC} 113$ on the polymer deposition rates. The maximum deposition rate can be attained at the two-one weight mixing ratio of $\mathrm{CFC113}$ and n-hexane in the flask tank. This deposition rate is 10 times as large as that of $n$-hexane itself. The mixing ratio of gas mixture 


\section{J. Photopolym. Sci. Technol., Vol.8, No.3, 1995}

in the reaction chamber may be different from the mixing ratio of monomers in the flask tank. The mixing ratio of the gas mixture in the reaction chamber were determined by the amount of evaporated gas from the flask tank. The amount of evaporated gases in the chamber were calculated by the decrease of residual liquid monomers in the flask tank, which were measured by gas chromatography. At a two-one mixing ratio ( $67: 33$ ) of $\mathrm{CFC} 113$ and n-hexane in the flask tank, the mixing ratio in the reaction chamber is calculated as about $70: 30$. As the weight mixing ratio is divided by each molecular weight, the molecular mixing ratio of CFC113 (M.W.187.5) and n-hexane(M.W.80) in the reaction chamber would be approximately $1: 1$.

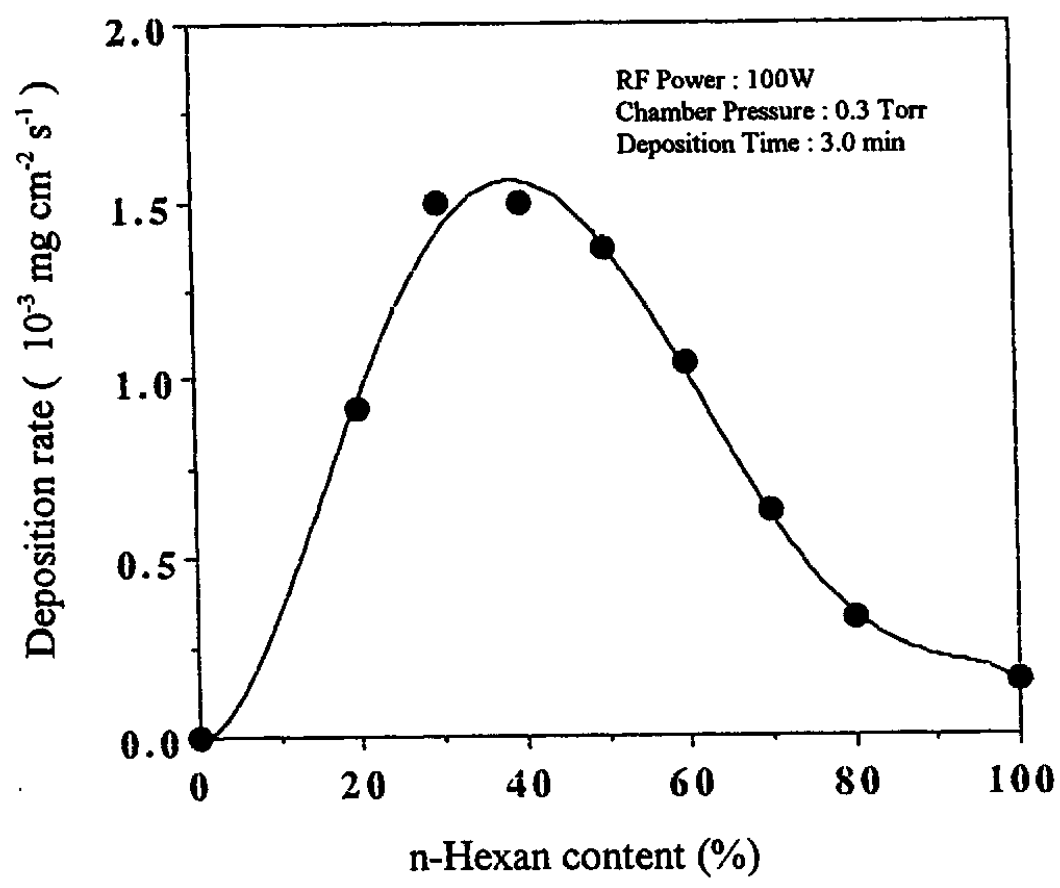

Fig. 2 The effect of n-hexan to $\mathrm{CFCl} 13$ on the polymer deosition rates

The solidification recovery was determined by the amount of deposition film on the whole top of lower electrode calculated by using the deposition rate on the cover glass. The percentage of the recovery was calculated by dividing the amount of deposition film by the amount of gas introduced into the chamber over three minutes time. The amount of gas introduced into the chamber was measured by weighing the decrease of the flask tank during the three minutes. At the maximum deposition rate, the solidification recovery is $16 \%$, which 


\section{J. Photopolym. Sci. Technol., Vol.8, No.3, 1995}

is higher than that of the CFC113 and ethylene mixture [3]. Polymerized material also existed in other places besides on the lower electrode, for example, on the upper electrode, the chamber wall and the lead lines. The total amount of collected polymeric materials from these places indicated that the recovery was about $50 \%$. If the amount ventilated from the chamber is considered, the recovery would be higher than $50 \%$.

\section{Characterization of the Deposition Film}

XPS spectrum of a deposition film polymerized at the condition of highest deposition rate is shown in Fig. 3. This result proved that the deposition film includes the elements of carbon, fluorine, chlorine and oxygen. XPS narrow spectrum of $\mathrm{C} 1 \mathrm{~s}$ is also seen in Fig. 4 indicating the existence of $\mathrm{C}-\mathrm{F}, \mathrm{C}-\mathrm{Cl}$ covalent bonds. IR spectrum shown in Fig. 5 finds absorption bands that can be assigned as $\mathrm{C}-\mathrm{F}, \mathrm{C}-\mathrm{Cl}, \mathrm{C}=0, \mathrm{C}=\mathrm{C}$ and $\mathrm{C}-\mathrm{H}$ vibrations. These results show that polymerized films from $\mathrm{CFC} 113$ and $\mathrm{n}$-hexane mixture contain not only carbon and hydrogen but also fluorine and chlorine. This means that some part of CFC113 was converted to a polymeric film. These films have a distinctive feature that $\mathrm{C}-\mathrm{H}$ bond in the films is much more than that of $\mathrm{CFC} 113$ and ethylene[3].

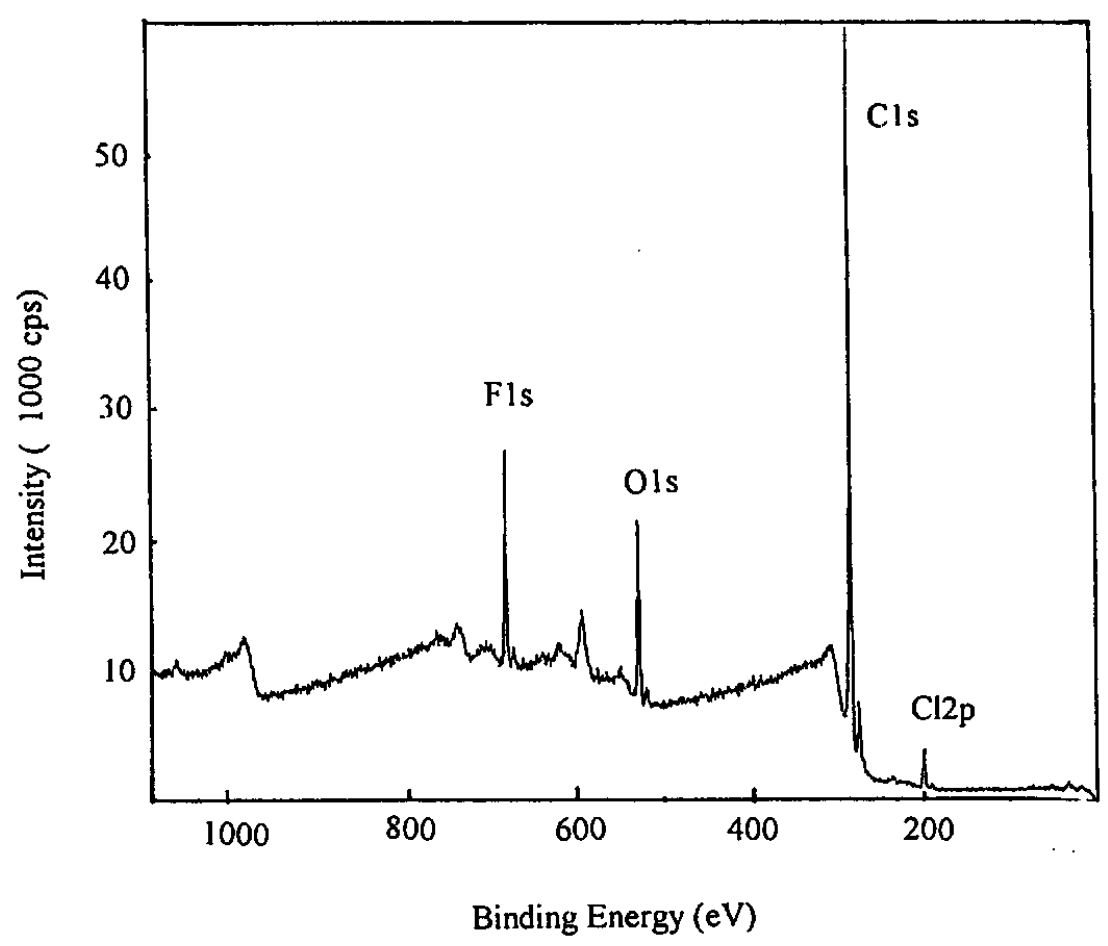

Fig. 3 XPS spectrum of a plasma polymerized film from $\mathrm{CFC113}$ and $\mathrm{n}$-hexane mixture 


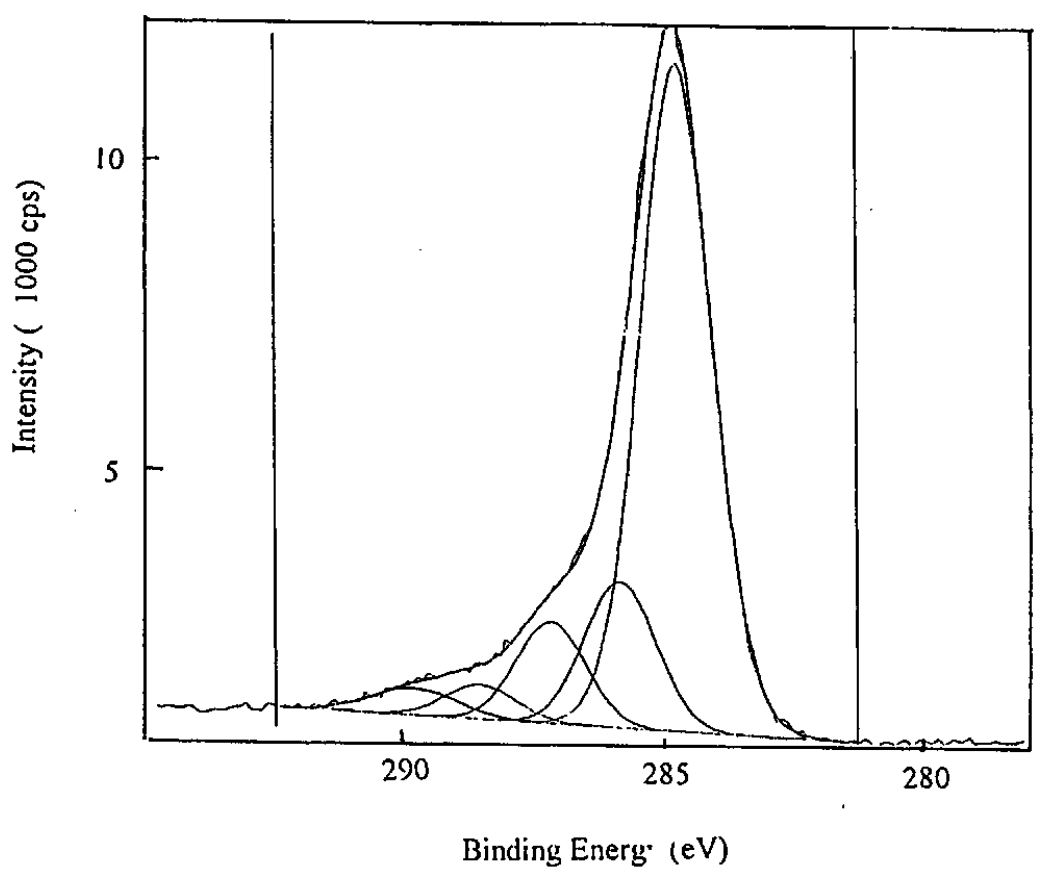

Fig. 4 XPS narrow spectrum of $\mathrm{Cls}$ of a plasma polymerized film from $\mathrm{CFC} 113$ and n-hexane mixture

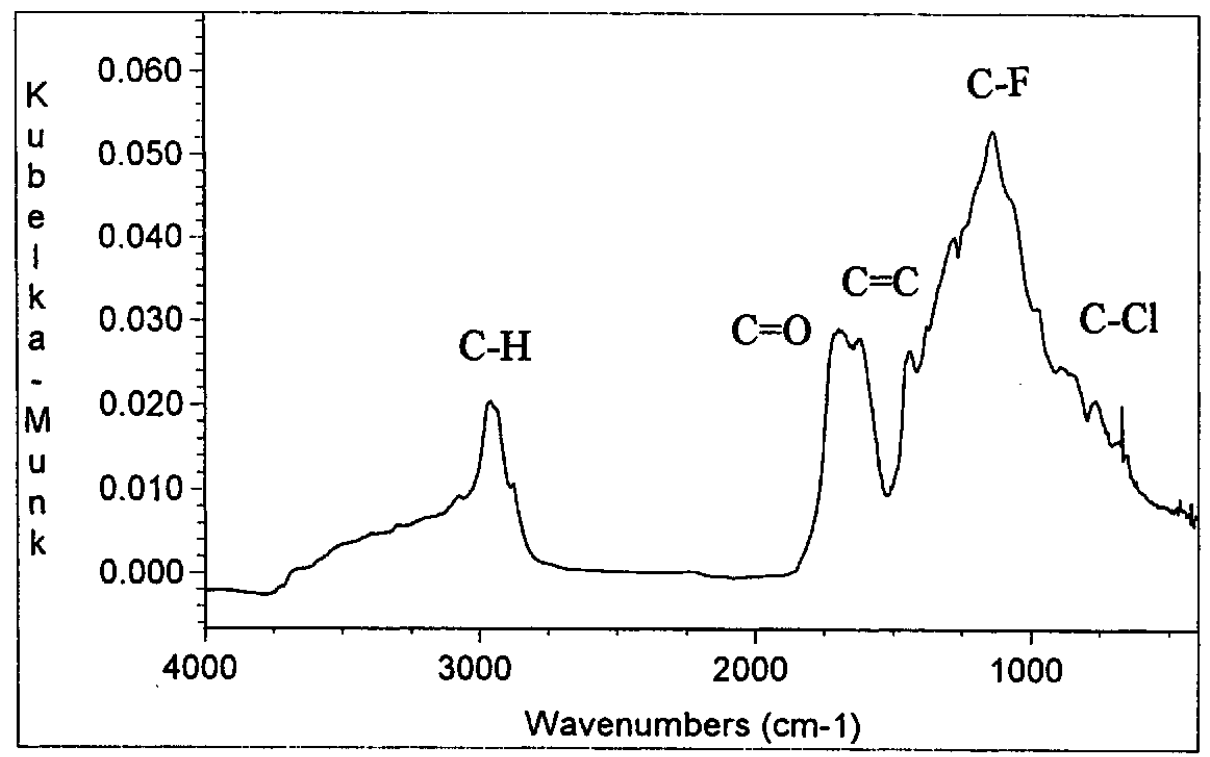

Fig. 5 IR spectrum of a plasma polymerized film from $\mathrm{CFC113}$ and n-hexane mixture

Table 1 shows $\mathrm{F} / \mathrm{C}$ and $\mathrm{Cl} / \mathrm{C}$ ratios, of the polymerized films, which were determined by XPS measurements, compared to the ratios of monomer gas mixtures. This table explains that about $30 \%$ of fluorine and $20 \%$ of chlorine were contained into polymeric film. Ion chromatography analysis of cold trapped substances detected some amount of Fluorine and chlorine ion. Some part of halogens in monomer were decomposed into ionic substances. 
Table $1 \mathrm{~F} / \mathrm{C}$ and $\mathrm{Cl} / \mathrm{C}$ ratios of the plasma polymerized filmsdetermined by XPS, compared to the ratios of monomer gas mixtures

Monomer gas mixture

(CFC113 : n-hexane)

weight molecular $\mathrm{F} / \mathrm{C}, \mathrm{Cl} / \mathrm{C}$

$\mathrm{F} / \mathrm{C}$ and $\mathrm{Cl} / \mathrm{C}$ ratios of

Plasma polymerized film

$\begin{array}{lllll}7: 3 & 1: 1 & 0.37 & 0.16 & 0.11 \\ 6: 4 & 1: 1.5 & 0.27 & 0.09 & 0.03 \\ 4: 6 & 1: 3.5 & 0.13 & 0.05 & 0.008 \\ 2: 8 & 1: 10 & 0.05 & 0.03 & 0.003\end{array}$

Fig. 7 shows UV-Visible spectra of the plasma polymerized films. The polymerized film of n-hexane added by $20 \%$ amount of CFC 113 provided a extremely different UV-VIS spectrum from the film of $\mathrm{n}$-hexane itself. The absorbance spectrum band positon shifted to a shorter wavelength side and the band width became broader. Fluorine atom contained in the film might cause the change of the electric state structure of the polymeric substance.

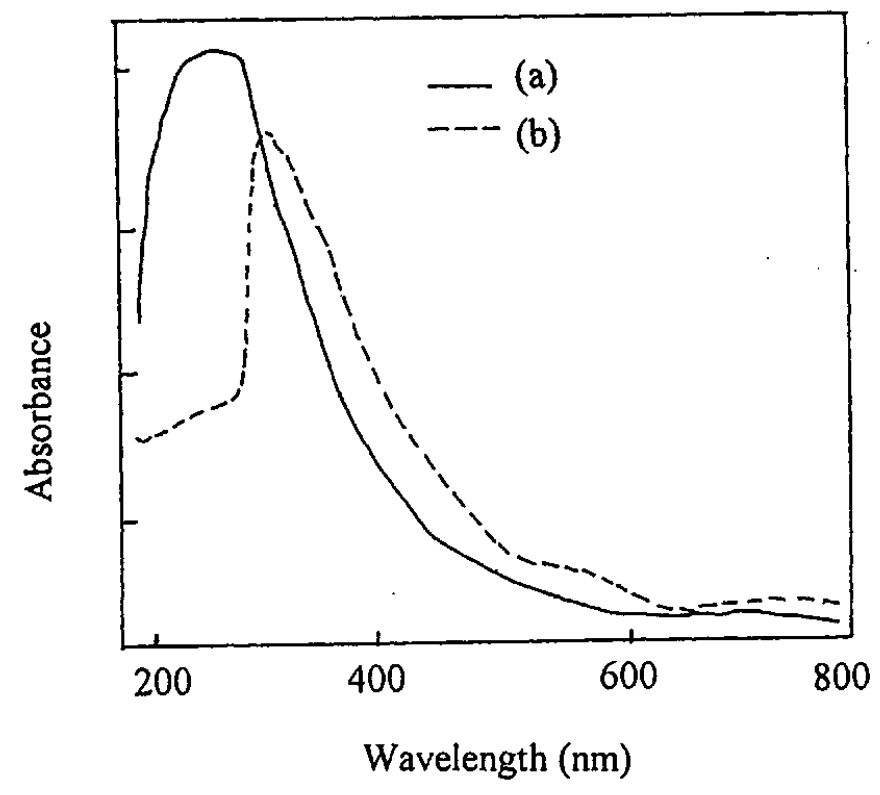

Fig. 6 UV-VIS spectra of plasma polymerized film ( monomer gas content;

(a) $\mathrm{CFC113} 20 \%+n$-hexane $80 \%$, (b) n-hexan $100 \%$ ) 
Studies on the plasma polymerization of fluorocarbon were reported [4],[5],[6]. The work was performed for potential applications in the field of the coating industry as a surface protective coating and functional films. The purpose of this plasma polymerization was originally to develop a new method of waste freon treatment. The plasma polymerization of CFC113 was attained at high deposition rate by the effect of $n$-hexane. These results provide the possibility to promote a method that converts specific freons to stable solid state substances. Furthermore, the polymerized films may possibly be developed for the reuse and resource of waste freon to functional films.

\section{Conclusion}

Plasma polymerization of chlorofluorocarbon was performed by RF cold plasma. Addition of n-hexane to $\mathrm{CFC} 113$ attained the deposition of plasma polymerized film effectively. Chlorine and fluorine were contained in the deposition films and bonded to carbon. one-one molecular gas mixing ratio of $\mathrm{CFCl13}$ and $\mathrm{n}$-hexane obtained the maximum deposition rate. The solidification recovery of the monomers was higher than $50 \%$. UV-Visible spectroscopic property of the films were significantly different from that of $n$-hexane itself.

\section{Acknowledgments}

The authors heartily acknowledge Mr. M. Takamura who measured XPS spectra.

\section{References}

1. T. Oda, T. Takahashi, H. Nakano, S. Masuda, IEEE Trans. Ind. Appl., 29 (1993) 787.

2. O. Kajiya, J. IEE Japan, 114 (1994) 719.

3. K. Miyashita, T. Ozawa, O. Tsuji, J. Chem. Soc. Japan, 12 (1994) 1140.

4. H. Yasuda, T. S. Hsu, J. Polym. Sci., 15 (1977) 2411.

5. K. Nakajima, A. T. Bell, M. Shen, J. Appl. Polym. Sci., 23 (1979) 2627.

6. A. Salhi, D. S. Soong, A. T. Bell, Polym. Eng. Sci., 21 (1981) 643 